\title{
Capsule Commentary on Belson et al., Effects of Discontinuation of Urate-Lowering Therapy: A Systematic Review
}

\author{
Mary A. Andrews, MD, MPH \\ Department of Medicine, Uniformed Services University, Bethesda, MD, USA.
}

$\mathrm{J}$ Gen Intern Med 33(3):334

DOI: $10.1007 / \mathrm{s} 11606-017-4251-3$

(c) Society of General Internal Medicine (outside the USA) 2017

\begin{abstract}
A ny physician who cares for patients with many comorbidities or aversion to pharmacologic therapy faces the conundrum of advising on the risks of therapy cessation in the context of limited evidence. This review by Belson et al. sheds some light on the risk of recurrent gouty arthritis or urolithiasis after cessation of urate-lowering (ULT) therapy by summarizing the results of eight observational studies of varying methodological quality. ${ }^{1}$ The recurrence rates for gouty arthritis ranged from $36.4 \%$ to $81 \%$, with a mean time to relapse of 15.8 to 56 months. The relapse rate for urolithiasis was only $15.1 \%$, although it was reported by only one study. The review is limited by the homogeneity of populations (overwhelmingly European men), lack of comparator groups, and the absence of recent data (only two studies published after 2000).

Two salient features of this review stand out. First, the range of time between cessation of ULT to first gouty episode is remarkably wide (4 to 96 months). Factors associated with relapse were investigated in only three studies, with trends towards increased risk in patients with unsurprising features such as higher serum urate levels, higher BMI, and longer duration of ULT. Further studies are needed to investigate these and other potentially unexplored factors of increased risk of gout recurrence after stopping ULT, with studies of behavioral modification (e.g., weight loss, moderation of animal protein and alcohol intake) of particular interest given the concurrent beneficial effects on comorbid conditions such as hypertension and heart disease. ${ }^{2}$
\end{abstract}

Second, while the risk of allopurinol toxicity in patients with advanced chronic kidney disease (CKD) is well known, ${ }^{3}$ this review reports a novel and potentially deleterious effect of allopurinol cessation in this population. The authors of one study found increased progression of CKD and worsening blood pressure control in patients with advanced CKD who were not treated with a renin-angiotensin system inhibitor (RASI), compared to those who were on such an agent. ${ }^{4}$ The potentially protective effects of ULT and modifying effect of RASI in patients with advanced CKD warrant further investigation, and physicians should carefully monitor blood pressure and renal function if stopping ULT in these patients.

Corresponding Author: Mary A. Andrews, MD, MPH; Department of MedicineUniformed Services University, Bethesda, MD, USA (e-mail: mary.andrews@usuhs.edu).

\section{Compliance with Ethical Standards:}

Conflict of Interest: The author has no conflicts of interest with any of the material in this manuscript.

\section{REFERENCES}

1. Belson V, Moreau P, Maruani A, Maisonneuve H, Giraudeau B, Fornier J-P. Effects of discontinuation of urate-lowering therapy: a systematic review. J Gen Intern Med. 2017. https://doi.org/10.1007/s11606-0174233-5.

2. Singh JA, Reddy SG, Kundukulam J. Risk factors for gout and prevention: a systematic review of the literature. Curr Opin Rheumatol. 2011;23(2): 192-202.

3. Stamp LK, Taylor WJ, Jones PB, et al. Starting dose is a risk factor for allopurinol hypersensitivity syndrome: a proposed safe starting dose of allopurinol. Arthritis Rheum. 2012;64(8):2529-36.

4. Talaat KM, El-Sheikh AR. The Effect of Mild Hyperuricemia on Urinary Transforming Growth Factor Beta and the Progression of Chronic Kidney Disease. Am J Nephrol. 2007;27(5):435-40. 